\title{
Nanoscale Measurement of Surface Roughness and the existing Surface Forces of Aluminum by AFM
}

\author{
Purna B Pun and Shobha K Lamichhane \\ Trivhuwan University, Depart of Physics, Prithivi Narayan Campus, Pokhara \\ puran_harpal@yahoo.com
}

\begin{abstract}
The surface contamination affects Atomic Force Microscope (AFM) performance. Thermal agitation during mapping doping, thermal oxidation, annealing impurities and crystal defects promotes the roughness, various kinds of forces on the surface can be detected by the interaction between tip of cantilever and sample. This interaction not only help us to understand the characteristics and morphology of the sample but also useful to measure the surface force of the aluminum sample too.
\end{abstract}

\section{Introduction}

Aluminum is a silvery white member of the boron group of chemical element. It is not soluble in water under normal circumstances. Aluminum is the third most abundant element, after oxygen and silicon. It makes up about $8 \%$ by weight of the Earth's solid surface [1]. Aluminum has very good ductility and the malleable property therefore, it has wide range of application in the present world in various field like in Building, in Transportation, in production Engineering, in Electronics, in Household etc [2].

In the last decade there has been an increasing interest in mesoscopic devices in which the size of the active elements is in nanoscale (between $1 \mathrm{~nm}$ and $100 \mathrm{~nm}$ ). Peculiar physical phenomena like the coulomb blockade and the quantization of the conductance emerge at this length scale, and their study is relevant to the design of novel nanoelectronic devices [3]. Surface roughness and surface force, can be analyzed by the AFM. In contact mode, tip of cantilever is scanned at a constant height. To prevent a risk that the tip collide with the sample surface and get damaged, feedback mechanism is employed to adjust the tip-to-sample distance to maintain a constant force between the tip and the sample. Now laser light from a solid state diode is reflected off the back of the cantilever and collected by a position sensitive detector (PSD) consisting of two closely spaced photodiode whose output signal is collected by a differential amplifier. From this output topography of the gives sample can be developed. The forces that are measured in AFM include van der Wall forces, capillary force, chemical bonding, electrostatic forces, magnetic forces, Casimir forces, salvation forces etc [4]. The topography along the X-Y plane, the observed van der Wall force against the cantileved deflection along the Z-axis (force distance curve) gives morphological, spectroscopical as well as dynamical behavior of the sample at the different molecular region.

\section{Experimental Details and Results \\ Calculation of the surface roughness}

Then aluminum sample is observed through the AFM.

Prepared sample is examined by AFM. What we found is that deflection of the cantilever is not uniform over the regions of the smaple surface [5]. Here, we take the root mean square roughness value which is given by the formula,

$$
R_{r m s}=\sqrt{\frac{\sum_{i=1}^{n}\left(Z_{i}-\bar{Z}\right)^{2}}{n}}
$$

Where $\mathrm{Zi}$ is the height of each data point, $\mathrm{Z}$ bar is the average of all height values in the image and $n$ is the number of data points within the image [6].The three dimensional image and the observed roughness of the given aluminum sample and retract mode are shown in figure 2 (a), 2 (b), Graph 2 (c) and Graph 2 (d). 


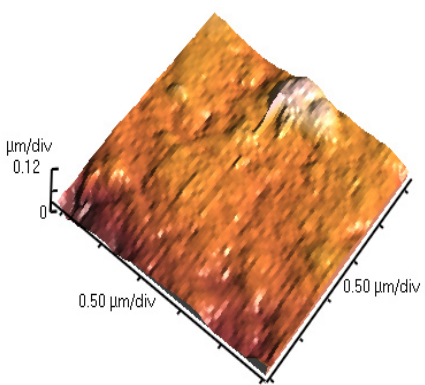

Fig.1 (a) 3D image of aluminum sample approach mode

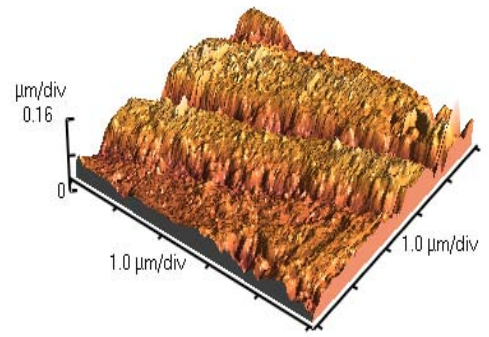

Fig.1 (b) 3D image of aluminum sample in in retract mode

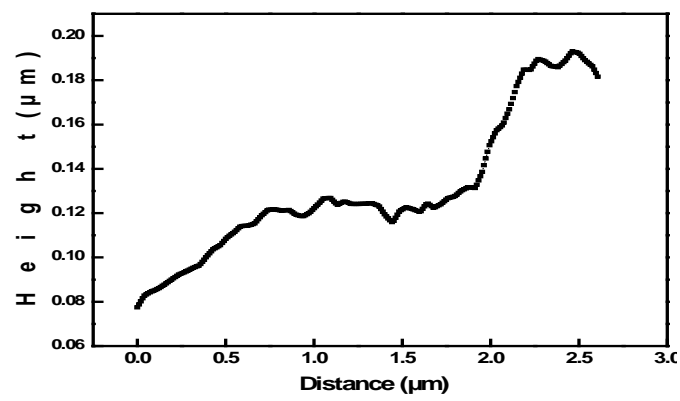

Fig. 1 (c) surface roughness of aluminum in approach mode

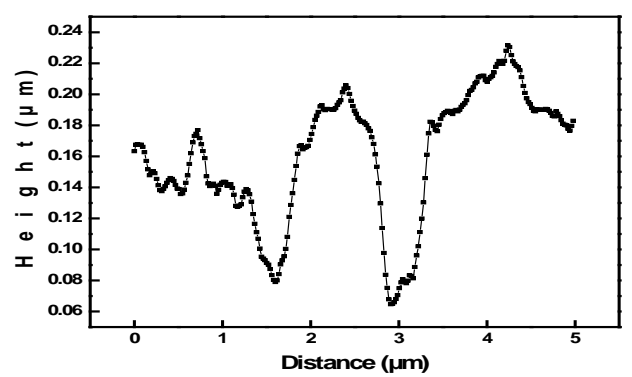

Fig. 1 (d) surface roughness of aluminum in contract mode

From the graph 1 (c) and 1(d), the calculated root mean square roughness of the aluminum in approach mode and retreat mode is found to be $R_{r m s 1} \cong R_{r m s 1} \cong 0.13$ Micrometer and $R_{r m s 2} \cong R_{r m s 2} \cong 0.15$ Micrometer respectively. Hence from the above calculation, we found that aluminum in approach mode have more smooth surface than in retract mode.

\section{Calculation of surface force (pull-off -force)}

When the tip of the cantilever is mounted on the surface of the sample, force curve is collected by monitoring vertical cantilever deflection and the interaction between tip of cantilever and sample .The cantilever deflection can be converted to applied force through the cantilever spring constant, $\left(\mathrm{k}_{\mathrm{N}}\right)$ by the equation 1 given by the Hook's law [7];

$\mathrm{F}_{\mathrm{N}}=\mathrm{K}_{\mathrm{N}} \Delta \mathrm{ZF_{ \textrm {N } }}=\mathrm{K}_{\mathrm{N}} \Delta \mathrm{Z} \ldots \ldots \ldots \ldots \ldots \ldots \ldots 1$

Let, if cantilever have the length (L), width (w), thickness ( $\mathrm{t}$ ) and the Young modulus (Y), then for the normal deflection, the spring constant of the cantilever is given by the equation 2[8].

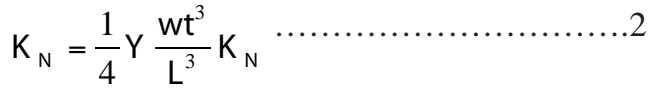

The work required to separate surface $1^{\text {st }}$ and surface $2^{\text {nd }}$ in the medium $3^{\text {rd }}$ is called the surface energy. It is also called work of adhesion and is related to the interfacial energies.

The work required separating surface $1^{\text {st }}$ and surface $2^{\text {nd }}$ in the medium $3^{\text {rd }}$ per unit area is given by equation 3 .

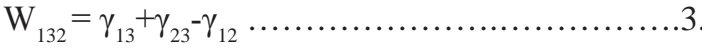

Where $\gamma$ is interfacial energy, 1 and 2 represents the two surfaces, and 3 refer to the contracting medium. When measurements are performed between symmetric contacts ( i. e., $\gamma_{1}=\gamma_{2}$ ) in medium 3 , equation 3 becomes.

$\mathrm{W}_{131}=2 \gamma_{18} \ldots \ldots \ldots \ldots \ldots \ldots \ldots \ldots .$.

If measurements are performed in a vacuum or in inert gas, the interfacial free energy $\gamma_{13}$ is simply the surface energy, $\gamma_{1}$. In this case, $\mathrm{W}_{132}$ reduces to $\mathrm{W}_{12}$, which represents the work of adhesion for an asymmetric contact and is equal to $\gamma_{1}+\gamma_{2}-\gamma_{12}$.

The adhesive force from DMT theory is given by

$\mathrm{F}_{\mathrm{ad}}=2 \pi \mathrm{RW}_{132}$ ..5.

Again the adhesive force given by the JKR theory is given by the equation;

$\mathrm{F}_{\mathrm{ad}}=1.5 \pi \mathrm{RW}_{132}$ .6.

These formulations provides a basis for relating the work of adhesion and the interfacial energies to the adhesion forces obtained by micro contact experiment i. e., the pull-off portion of 
Vol. II May 2011

the force. The force curve can be divided into several regions as shown in Figure 2 (e).

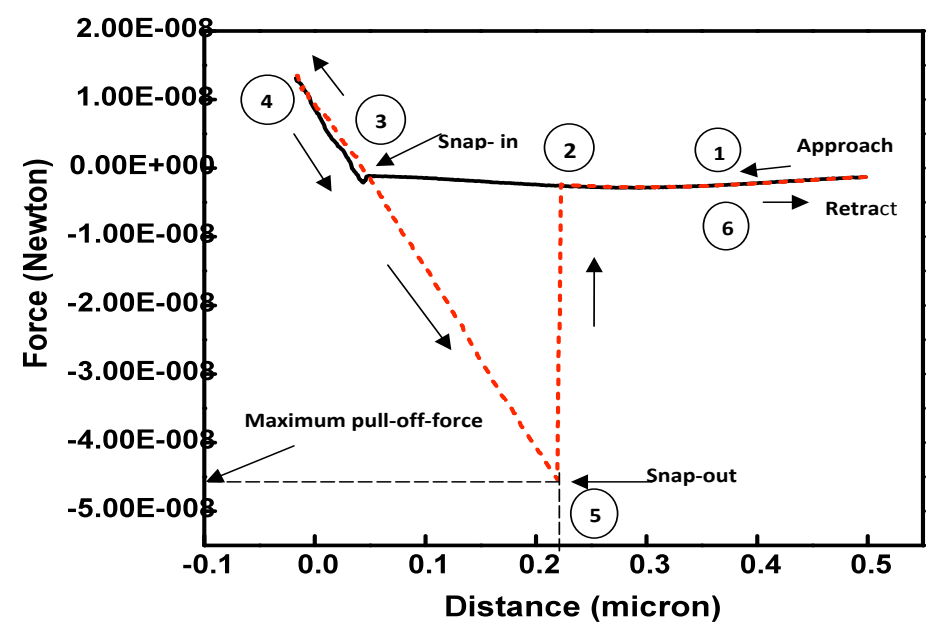

Figure 1 (e) Force Vs Distance Curve of the Aluminum Sample

$$
\begin{aligned}
& \text { The tip sample is sufficiently separated such that there is } \\
& \text { no detectable interaction. At this distance cantilever is in } \\
& \text { its no interacting equilibrium position. } \\
& \text { As the separation distances decreases, various long range } \\
& \text { and the short range forces come into existance which are } \\
& \text { given in the table below. }
\end{aligned}
$$

\begin{abstract}
As the separation distances decreases, various long range and the short range forces come into existance which are given in the table below.
\end{abstract}

Table 1. Types of Interaction Forces and Distances.

\begin{tabular}{|l|l|l|}
\hline \multicolumn{1}{|c|}{ Type } & \multicolumn{1}{|c|}{$\begin{array}{c}\text { Distances } \\
(\mathrm{nm})\end{array}$} & \multicolumn{1}{c|}{ Note } \\
\hline $\begin{array}{l}\text { Long range } \\
\text { Electrostatic force in } \\
\text { airDouble layer force in } \\
\text { electrolyte solution Van } \\
\text { der Wall force }\end{array}$ & $\begin{array}{l}100 \\
100\end{array}$ & $\begin{array}{l}\text { Depends on } \\
\text { electrolyte } \\
\text { concentration }\end{array}$ \\
\hline $\begin{array}{l}\text { Short range } \\
\text { Surface induced solvent } \\
\text { ordering Hydrogen } \\
\text { bonding force contact }\end{array}$ & 5 & $\begin{array}{l}\text { Depends on the } \\
\text { molecular size of } \\
\text { the solvent }\end{array}$ \\
\hline
\end{tabular}

These forces include attractive/repulsive electrostatic interaction, which are the result of the electrical double layer formed in aqueous electrolyte, and van der Wall interactions. At very small separations in liquid media, surface-induced solvent ordering may be detected.

Nanoscale Measurement of Surface Roughness and the existing...)

(3)

Figure 1 (e) represents an ideal force curve when an adhesive contact is formed between the tip and sample (e. g., adhesion due to capillary farces when operating in air)[9].

From the Figure 1 (e) the maximum value of the pull-force of aluminum sample is found experimentally as

\section{Pull-off force, $F_{\text {pull-off }}=-4.50 \mathrm{e}^{-8.08}$ Newton}

\section{Conclusion}

In AFM experiment, the cantilever deflection is very sensitive for the measurement of the topography and the interaction between tip and sample.Cantilever deflection can also depends on the surface roughness of the sample, more the surface roughness more will be the deflection and vice versa. The adhesion force or the maximum pull-off force is inversely proportional to the sixth power of cantilever deflection or the tip-sample separation and experimentally calculated value is Pull-off force, $\mathbf{F}_{\text {pull-off }}=\mathbf{- 4 . 5 0}$ $\mathbf{e}^{-8.08}$ Newton equilibrium distance (at which all the forces balances each other and there is no any forces affecting the cantilever deflection) is approximately found to $\mathbf{0 . 2 2}$ micron. Hence certain laws of the physics (force distance curve) can be verified experimentally. 


\section{Reference}

1. http://en.wikipedia.org/wiki/Aluminium

2. http://sam.davyson.com/as/physics/aluminium/siteus/ structure.html

3. Andrea Notargiacomo, Vittorio Foglietti and Florestano Evangelisti (1995),"Sub-100 nm Patterning of Aluminum Film by AFM Local Oxidation", page-1, Istituto di Elettronica dello Stato Solido (IESS) - CNR, Via Cineto Romano 42

4. http://www.tcm.phy.cam.ac.uk/ cjp20/old/lectures/ topic1.pdf

5. Lamichhane, S.K; 2005. " MEMS: Response of a strained silicon semiconductor structure" page -6 ,Ph. D. Thesis , JNU ,New Delhi.

6. Brin R. Liwandowski, 2004, Scanning Probe Microscopy Investigations of (1)Arrays of cysteine-coated cds nanoparticles and (2)Structures formed during the early stages of the corrosion of Copper surfaces", page41, Louisiana State University and Agricultural and Mechanical College.

7. Hajime Takona, Jeremy R. Kenseth, Sze-Shu Wong, Janese C. O'Brien, and Marc D. Porter, 1999. "Chemical and Biochemical Analysis Using Scanning Force Microscopy," Vol.-99,No.-10,page-2847, Ames Laboratory-USSOE, Microanalytical Instrumentation Center, and Department of Chemistry, lowa.

8. http://en.wikibooks.org/wiki/Nanotechnology/AFM

9. Hajime Takona, Jeremy R. Kenseth, Sze-Shu Wong, Janese C. O’Brien, and Marc D. Porter, 1999,"Chemical and Biochemical Analysis Using Scanning Force Microscopy," Vol.-99,No.-10,page-2859, Ames Laboratory-USSOE, Microanalytical Instrumentation Center, and Department of Chemistry, lowa. 